\title{
Lidocaine Infusion Improves the Functionality of Intraoperative Nerve Monitoring During Thyroid Surgery: A Prospective, Randomized, Double-Blinded Study
}

\author{
Ramasamy Govindarajan a, h, Ajay Shah ${ }^{\mathrm{b}}$, Saiganesh Ravikumar ${ }^{\mathrm{b}, \mathrm{c}}$, Sunil K. Reddy, d, \\ Umashankkar Kannan $^{\text {b, e }}$, Amar N. Mukerji ${ }^{b}$, f, Jasmine G. Cherian', \\ Crista Foster ${ }^{\mathrm{a}}$, Dave Livingstone ${ }^{\mathrm{a}}$
}

\begin{abstract}
Background: Intraoperative nerve monitoring (IONM) to assess the recurrent laryngeal nerve function during thyroid surgery is becoming the standard of care across many institutions. The successful deployment and data analysis from the IONM require complete laryngeal relaxation and reflex suppression. We investigated the role of intravenous lidocaine infusion (IVLI) to provide such operating conditions, under a lighter plane of anesthesia and fewer hemodynamic fluctuations.
\end{abstract}

Methods: Sixty-five patients were randomly assigned to lidocaine group (LG) or placebo group (PG) based on the computer-generated coding developed by the pharmacy department. The study medication (SM) was delivered by the pharmacist in a pre-filled coded syringe to the investigator $30 \mathrm{~min}$ prior to the surgery. All the patients were anesthetized by narcotic and inhalation based general anesthesia. The SM was administered at the rate of $1.5 \mathrm{mg} / \mathrm{kg} / \mathrm{h}$ following a loading dose of $1 \mathrm{mg} / \mathrm{kg}$. Dragonfly ${ }^{\circledR}$ laryngeal surface electrode and Nerveana ${ }^{\circledR}$ nerve locator system were used for IONM during surgery.

Results: The proportion of patients requiring lower strength stimulating current (StMC) at $0.5 \mathrm{~mA}$ was significantly higher in the $\mathrm{LG}$

Manuscript submitted March 9, 2021, accepted March 22, 2021

Published online April 27, 2021

aDivision of North American Partners in Anesthesia, Department of Anesthesia, BronxCare Health System, 1650 Grand Concourse, Bronx, NY 10457, USA

${ }^{b}$ Department of Surgery, BronxCare Health System, 1650 Grand Concourse, Bronx, NY 10457, USA

${ }^{\mathrm{c}}$ Current Affiliation: University of Rochester School of Medicine, 601 Elmwood Ave., Rochester, NY 14642, USA

${ }^{\mathrm{d} C u r r e n t ~ A f f i l i a t i o n: ~ P a r k v i e w ~ R e g i o n a l ~ M e d i c a l ~ C e n t e r, ~} 11104$ Parkview Plaza Drive, Fort Wayne, IN 46845, USA

${ }^{\mathrm{e}}$ Current Affiliation: Florida Surgical Specialists, Bradenton, FL 34208, USA ${ }^{\mathrm{f} C u r r e n t}$ Affiliation: Heartland Regional Medical Center, Marion, IL, USA gDepartment of Pharmacy, BronxCare Health System, 1650 Grand Concourse, Bronx, NY 10457, USA

${ }^{\mathrm{h}}$ Corresponding Author: Ramasamy Govindarajan, Department of Anesthesia, Division of North American Partners in Anesthesia, 1650 Grand Concourse, Bronx, NY 10457, USA. Email: ramasgovi@hotmail.com

doi: https://doi.org/10.14740/jocmr4458 than in the PG $\left(X^{2}(1, N=61)=10.1615, P=0.001434\right)$. Similarly, the proportion of patients with the drop in the aggregate impedance level (DAIL) by $<50 \%$ at the end of surgery was significantly higher in the $\mathrm{LG}$ than in the PG $\left(\mathrm{X}^{2}(1, \mathrm{~N}=61)=15.982, \mathrm{P}=0.000064\right)$. In addition, the proportion of patients with the hypotensive episodes requiring rescue medications more than twice during surgery was significantly lower in the LG than in the PG $\left(\mathrm{X}^{2}(1, \mathrm{~N}=61)=0.0183\right.$, $\mathrm{P}<0.05)$.

Conclusions: The enhanced laryngeal relaxation and the reflex suppression afforded by the IVLI could have enabled a lower StMC to elicit a positive signal. The lower StMC promotes less intense laryngeal alterations as evidenced by the lower DAIL in the LG. IVLI can enhance the functionality of the IONM during prolonged operating time and the resultant increased number of IONM stimulations, while providing a stable hemodynamic environment.

Keywords: IONM; Lidocaine infusion; Recurrent laryngeal nerve monitoring; Thyroid and parathyroid surgery

\section{Introduction}

The use of intraoperative nerve monitoring (IONM) in combination with visual recurrent laryngeal nerve (RLN) identification results in significant lower postoperative RLN palsy rates than RLN identification without IONM [1,2]. The alerting of the surgeon early to potential RLN problems is a pre-requisite for the decrease of RLN injury and should result in lower RLN palsy rates and an improved quality of life for patients [3]. The decrease in the identification time of the RLN, while employing IONM during the thyroid surgery, has been documented in the literature [4]. Because of the simplicity of design and cost effectiveness, recording of electromyography of the vocal cords using surface electrodes integrated or attached to the endotracheal tube (ETT) is perhaps the most widely used technique of the IONM [5]. Complete functionality of the IONM depends on near total relaxation and reflex suppression without the use of neuromuscular blocking agents (NMBAs) [5]. The depth of anesthesia required to meet that criteria often leads to hemodynamic instability [5]. Intravenous (IV) lidocaine has 
been widely employed in anesthesia to suppress laryngotracheal reflexes, intubation-induced bronchospasm and to blunt adverse cardiovascular response during intubation. Interpretation of the data from the IONM during surgery depends on the near perfect positioning of the laryngeal electrodes and the near total suppression of the laryngeal reflexes. We investigated the role of IV lidocaine infusion (IVLI) in improving the functionality of IONM during thyroid and parathyroid surgery in this prospective, randomized, double-blinded, placebo-controlled study.

\section{Materials and Methods}

\section{Subjects}

This study was approved by the Institutional Review Board of the Bronx Lebanon Hospital Center, Bronx, NY, USA and the protocol was registered with the ClinicalTrials.gov Registry as NCT02479789. It was conducted in compliance with the ethical standards of our institution on human subjects as well as with the Helsinki Declaration. Patient recruitment was initiated during the preoperative visit to the surgical clinic. We enrolled 65 male and female patients aged 18 - 85 years scheduled for thyroid and parathyroid surgery including redo surgery with American Society of Anesthesiologists (ASA) physical status classification I to III. Patients undergoing additional procedures, known hypersensitivity to lidocaine and at high risk for IV lidocaine use due to congestive heart failure and arrhythmia were excluded from the study.

\section{Study design}

This study was a double-blinded, placebo-controlled, randomized, quadruple masked (participant, care provider, investigator and outcome assessor) clinical trial. Participants were recruited from September 2015 to September 2018 and randomly assigned to either the lidocaine group (LG) or the placebo group (PG). The study medication (SM) was delivered to the operating room $30 \mathrm{~min}$ prior to the commencement of the surgery by the pharmacy department, in a pre-filled syringe labelled with a computer-generated code.

The randomization list was generated by the pharmacy staff in blocks based on computer coding. Study group assignment was concealed from the patients, clinicians and research staff until after all the study data were collected and documented for the last patient.

\section{Outcome measures}

The primary outcome measures were the suppression of laryngotracheal reflexes during thyroid and parathyroid surgery, the time-frame being from the beginning to the end of surgery. We evaluated the laryngotracheal activity as a function of maintenance of contact between the laryngeal electrodes and vocal cords by documenting the lowest level of stimulating current
(StMC) to elicit a positive signal and the drop in aggregate impedance level (DAIL) by $<50 \%$ at the conclusion of the surgery. Intraoperative monitoring of the laryngotracheal activity was objectively recorded and evaluated from the data from the IONM (StMC and DAIL).

The secondary outcome measures included the amount of anesthesia medications used, postoperative pain control, pain medications required, nausea and overall patient comfort. The total amount of anesthesia medications used, and postoperative pain medications required were recorded in absolute numbers. The time-frame for the postoperative monitoring started on arrival to the post anesthesia care unit (PACU) and ended at $24 \mathrm{~h}$ after surgery. Postoperative pain control, postoperative nausea and overall patient comfort were recorded on a scale of $1-10$.

\section{Statistical analysis}

Data were managed and analyzed using the SAS 9.3 statistical software (Cary, NC, USA). We used Chi-square test (Fisher's exact test when the value in one of the cells was $<5$ ) for categorical variables and unpaired two sample $t$-test for continuous variables to test the difference between the two study groups. A P-value of less than 0.05 was considered statistically significant.

\section{Perioperative management}

The IONM setup was prepared by applying Dragonfly ${ }^{\circledR}$ Single Channel Laryngeal Surface Electrode (Electrode LSE 500Ms; Neurovision Medical Products, Ventura, CA, USA) to a \#7 cuffed ETT (Medline Industries, Mundelein, IL, USA) according to the manufacturer's instructions. The nerve locator system was tested as per their instructions.

When the patient was received in the operating room, in addition to standard ASA monitors, a radial arterial line was placed under local analgesia for closer hemodynamic monitoring. Lidocaine $1 \mathrm{mg} / \mathrm{kg}$ was administered as an IV bolus for all the patients prior to induction. The SM received from the pharmacy in prefilled coded syringe was set up to deliver at the volume equivalent of $1.5 \mathrm{mg} / \mathrm{kg} / \mathrm{h}$ through an infusion pump. The patients were preoxygenated and induced with a bolus of midazolam $2 \mathrm{mg}$ and fentanyl $1.25 \mu \mathrm{g} / \mathrm{kg}$ followed by propofol $1.5 \mathrm{mg} / \mathrm{kg}$ and cisatracurium $4 \mathrm{mg}$. Intubating conditions were established by succinylcholine $1.2 \mathrm{mg}$ IV bolus and the airway was secured with the pre-prepared surface electrode mounted ETT by direct vision laryngoscopy. Anesthesia was maintained with air, oxygen and sevoflurane at the desired minimum alveolar concentration levels titrated to effect. The placement of the electrode plates appropriately in contact with the vocal cords was verified with a Glidescope ${ }^{\circledR}$ after final positioning the patient with the desired neck extension. A bispectral index (BIS) monitor (Model 1 A 2000; Aspect Medical Systems, Newton, MA, USA) was connected to guard against "recall" during lighter planes of anesthesia and a train of four (TOF) monitor (Microstim Plus, Neurotechnology, Houston, TX, USA) was employed to guard against inadvertent neuro- 


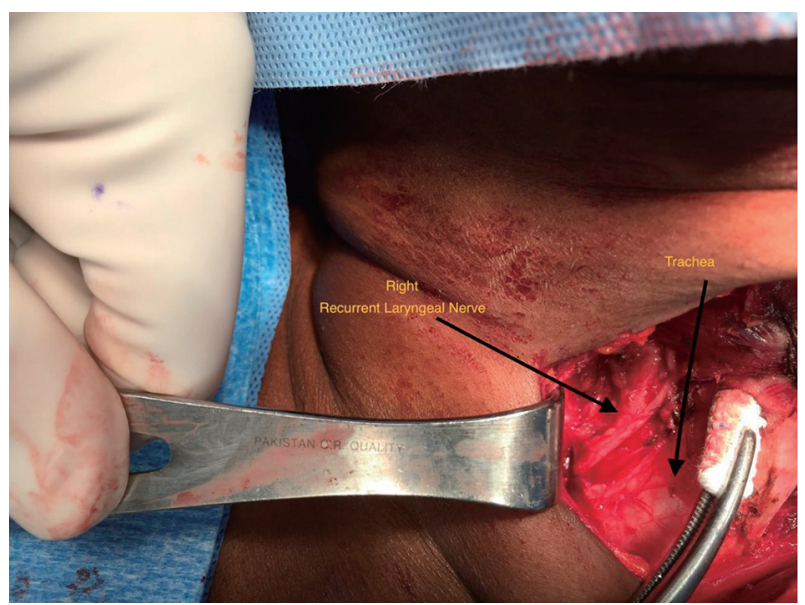

Figure 1. Right recurrent laryngeal nerve dissection.

muscular blockade (NMB). Hemodynamic fluctuations during surgery were treated with $\beta$-blockers and sympathomimetic agents as appropriate. The initial stimulation level was set at $1.5 \mathrm{~mA}$ and gradually reduced to $0.5 \mathrm{~mA}$. The lowest strength of the StMC required to elicit a positive signal upon the RLN stimulation was the goal, while the visual confirmation of the RLN was the gold standard for the study. The DAIL at the end of surgery was recorded. Greater than 50\% DAIL was considered significant indicator of loss of contact between the electrode sensors and vocal cords. Inability to elicit a signal at $2 \mathrm{~mA}$ was considered as equipment/placement failure. Additional doses of fentanyl were administered as needed to sustain basal narcosis. The SM infusion rate was tapered down and discontinued upon extubation.

Meticulous dissection and visual identification of the RLN were the primary focus during surgery (Figs. 1 and 2). Mobilizing the thyroid lobe medially until cricothyroid joint is palpable served as the initial starting point for the RLN location. The next step was to define the trachea esophageal groove (TEG) by lateral traction on the paratracheal tissue around the carotid and medial traction on the trachea. Identification of the inferior thyroid artery in the TEG serves as an additional guide to the RLN. Definitive confirmation of the RLN identity was by the IONM stimulation of the nerve resulting in the palpable and visual contraction of the posterior cricoarytenoid muscle. The number of stimuli delivered until confirmatory cricoarytenoid contraction depended upon the level of resident participation. The attending surgeon's experience predated the IONM by many years, while residents needed frequent reassurances approaching the RLN and the number of IONM stimulations varied accordingly.

The patients stayed overnight in the PACU for observation. Their vital signs and study parameters were recorded at appropriate intervals. PACU data were determined and documented by the PACU staff not involved with the study.

\section{Results}

While we recruited 65 patients for this study, we were able to

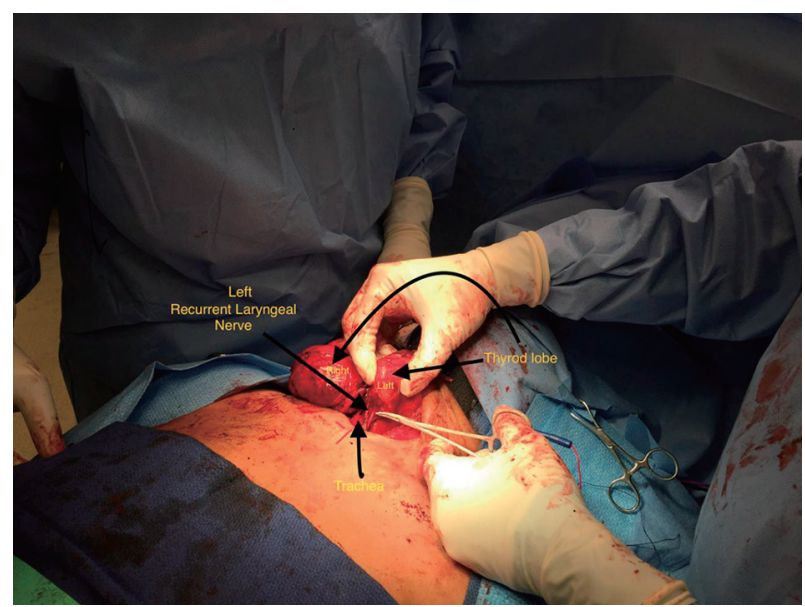

Figure 2. Left recurrent laryngeal nerve dissection.

use 61 patients $(93.8 \%)$ in the final analysis due to missing core data points.

\section{Study population characteristics}

The study population were of mostly women ( $\mathrm{N}=54,88.5 \%)$, Hispanic $(\mathrm{N}=59,96.7 \%)$ with a mean age of 58 (standard deviation $(\mathrm{SD})=9.6$ ) and a mean body mass index of 29.4 (SD $=4.8$ ). Most of the patients in the study population were not current smokers $(\mathrm{N}=55,90.2 \%)$. About a quarter of patients $(\mathrm{N}=15,24.6 \%)$ had three or more comorbid conditions such as hypertension, diabetes mellitus, hyperlipidemia, along with coronary artery disease or chronic obstructive pulmonary disease or hypothyroidism. Over a half $(\mathrm{N}=33,54.1 \%)$ of the patients had surgery for thyroid adenoma, nearly a quarter $(\mathrm{N}=$ $13,21.3 \%)$ for thyroid carcinoma, about a tenth $(\mathrm{N}=6,9.8 \%)$ for parathyroid adenoma and the rest $(\mathrm{N}=9,14.8 \%)$ for other reasons such as a combination of thyroid/parathyroid pathology or Hashimoto's/chronic lymphocytic thyroiditis (Table 1).

\section{Primary outcome measures}

The proportion of patients that required a lower strength StMC at $0.5 \mathrm{~mA}$ (manufacturer's recommended strength is $1.5 \mathrm{~mA}$ ) was significantly higher in the LG than in the PG $\left(X^{2}(1, N\right.$ $=61)=10.1615, \mathrm{P}=0.001434)$. Similarly, the proportion of patients with the DAIL by $<50 \%$ at the end of surgery was significantly higher in the LG than in the PG $\left(\mathrm{X}^{2}(1, \mathrm{~N}=61)=\right.$ 15.9816, $\mathrm{P}=0.000064$ ) (Fig. 3).

\section{Secondary outcome measures}

Secondary outcome measures failed to achieve statistical significance.

\section{Exploratory outcomes}


Table 1. Selected Characteristics of Study Population

\begin{tabular}{|c|c|c|c|}
\hline & \multicolumn{3}{|c|}{ Study group } \\
\hline & All, N (\%) & Lidocaine, N (\%) & Placebo, N (\%) \\
\hline Total & $61(100.0)$ & $32(100.0)$ & $29(100.0)$ \\
\hline Male & $7(11.5)$ & $2(6.3)$ & $5(17.2)$ \\
\hline Female & $54(88.5)$ & $30(93.8)$ & $24(82.8)$ \\
\hline Black & $2(3.3)$ & $1(3.1)$ & $1(3.4)$ \\
\hline \multicolumn{4}{|l|}{ Age } \\
\hline Mean & 57 & 56 & 58 \\
\hline Standard deviation & 9.6 & 9.5 & 9.6 \\
\hline \multicolumn{4}{|l|}{ Current smoker } \\
\hline Yes & $6(9.8)$ & $3(9.4)$ & $3(10.3)$ \\
\hline No & $55(90.2)$ & $29(90.6)$ & $26(89.7)$ \\
\hline \multicolumn{4}{|l|}{ Co-morbidity* } \\
\hline 0 condition & $16(26.2)$ & $8(25.0)$ & $8(27.6)$ \\
\hline 1 conditions & $20(32.8)$ & $14(43.8)$ & $6(20.7)$ \\
\hline 2 conditions & $10(16.4)$ & $4(12.5)$ & $6(20.7)$ \\
\hline$\geq 3$ conditions & $15(24.6)$ & $6(18.8)$ & $9(31.0)$ \\
\hline \multicolumn{4}{|l|}{ Pathology } \\
\hline
\end{tabular}

${ }^{*} 0$ condition: no comorbidity; 1 condition: hypertension or diabetes mellitus or hyperlipidemia or chronic obstructive pulmonary disease; 2 conditions: hypertension as well as hyperlipidemia or chronic obstructive pulmonary disease; $\geq 3$ conditions: hypertension, diabetes mellitus, hyperlipidemia, as well as coronary artery disease or chronic obstructive pulmonary disease or hypothyroidism.

Although originally was not planned as a part of the study, there were few additional outcomes that were significantly advantageous for the patients in the LG which we thought were worth reporting. For example, the diastolic blood pressure at extubation was significantly lower among LG patients $(\mathrm{M}=$ 77.3, $\mathrm{SD}=8.5)$ compared to those in the $\mathrm{PG}(\mathrm{M}=82.0, \mathrm{SD}=$ $8.9)(\mathrm{t}(59)=-2.7, \mathrm{P}=0.04)$. The total duration of surgery was also significantly reduced among the patients in the LG compared to the patients in the PG. While over a three quarters of the patients in the LG required $\leq 120$ min for surgery, only less than a half of the patients in the PG needed a surgical duration of $\leq 120 \min \left(\mathrm{X}^{2}(1, \mathrm{~N}=61)=6.7, \mathrm{P}=0.009\right)$. The proportion of patients that required additional cisatracurium in the LG was significantly lower than that in the PG $\left(X^{2}(1, N=61)\right.$
$=8.8, \mathrm{P}=0.002)$. Similarly, the proportion with hypotensive episodes requiring rescue medications more than twice during surgery was significantly lower in the LG than in the PG $\left(\mathrm{X}^{2}\right.$ $(1, \mathrm{~N}=61)=0.0183, \mathrm{P}=0.02)($ Table 2$)$.

\section{Discussion}

Electrical stimulation of the RLN during thyroid surgery was studied and reported as early as 1969 by Flishberg and Lindholm [6]. Advances in electrophysiological monitoring, electrode technology, recording and stimulation technics and monitoring equipment have made monitoring of the RLN a practical tool for the surgeons [7]. 


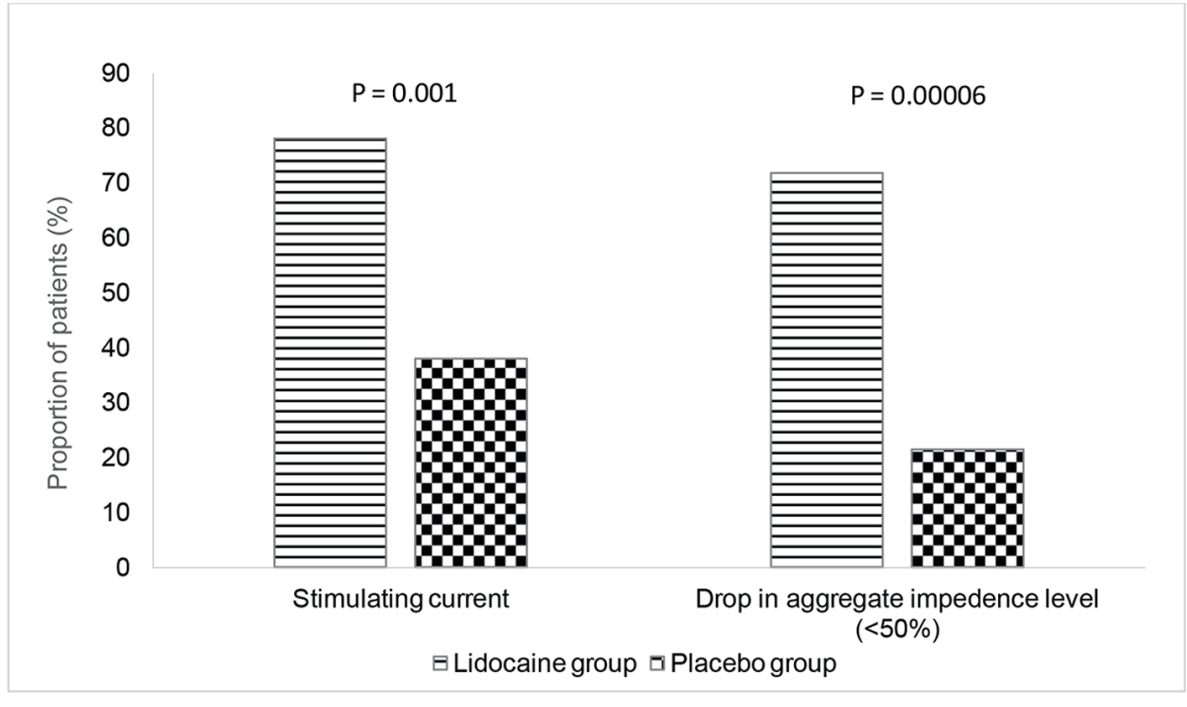

Figure 3. Primary outcome measures.

The summed action potentials (SAcPs) of the vocalis muscle have been measured intraoperatively to evaluate the RLN function during surgery. The absence of these potentials is a sign of permanent or temporary paralysis of the RLN. General anesthesia and muscle paralysis have the potential to decrease or even abolish these potentials [8].

The most dreaded complication following thyroid and to a lesser extent parathyroid surgery is the RLN palsy. Although the overall incidence of nerve palsy is low, when it occurs it is a devastating lifelong handicap $[9,10]$. Experienced surgeons can also injure the nerve, resulting in persistent nerve palsy in $1-2 \%$ of patients [4]. Despite the advances made over the years on neuromonitoring, atraumatic dissection and visual identification remain the gold standard in RLN preservation. The nerve is highly sensitive and even a little tension on it can result in temporary paresis [8]. In addition to the atraumatic dissection of the RLN, intraoperative neuromonitoring is superior to surgical exposure (of the nerve) alone, as not only the morphological but also the functional integrity can be demonstrated [8]. Visualization of an intact RLN and positive stimulation appear to ensure subsequent nerve function [11]. Positive stimulation is associated with a favorable medium-term and long-term prognosis $[12,13]$. The RLN must be stimulated as inferiorly as possible, as stimulation too close to its site of entry may fail to detect a more inferior lesion [11]. Absence of visualization of the RLN was corre-

Table 2. Exploratory Outcome Measures

\begin{tabular}{|c|c|c|c|c|c|}
\hline \multirow{2}{*}{ Exploratory outcome } & \multicolumn{2}{|c|}{ Study group } & \multirow{2}{*}{ t-value } & \multirow{2}{*}{$\mathrm{X}^{2}$ value } & \multirow{2}{*}{ P value $^{\mathrm{a}}$} \\
\hline & Lidocaine, $\mathbf{N}(\%)$ & Placebo, N (\%) & & & \\
\hline Total & $32(100.0)$ & $29(100.0)$ & - & - & - \\
\hline \multicolumn{6}{|c|}{ Diastolic blood pressure $(\mathrm{mm} \mathrm{Hg})^{\mathrm{b}}$} \\
\hline Mean & 77.3 & 82.8 & -2.7 & N/A & 0.043 \\
\hline Standard deviation & 8.5 & 8.9 & & & \\
\hline$\leq 120$ & $25(78.1)$ & $13(44.8)$ & N/A & 6.7 & 0.009 \\
\hline$>120$ & 7 (21.9) & $16(55.2)$ & & & \\
\hline \multicolumn{6}{|l|}{ Cisatracurium $(2-4 \mathrm{mg})^{\mathrm{c}}$} \\
\hline Yes & $11(34.4)$ & $21(72.4)$ & N/A & 8.8 & 0.002 \\
\hline No & $21(65.6)$ & $8(27.6)$ & & & \\
\hline
\end{tabular}

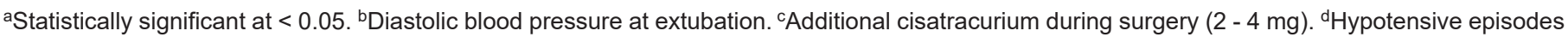
requiring rescue medications more than twice during surgery. $X^{2}$ : Chi-square; N/A: not applicable. 
lated with a high rate of RLN paralysis [11]. Cancer surgery, Graves' disease, redo surgery, bilateral RLN dissection, large thyroid lobe with intrathoracic extension and a history of irradiation or inflammation have all been associated with higher risk of RLN paralysis. Parathyroidectomy was associated with a low risk of RLN paralysis due to the very limited traction on the RLN during dissection [11]. Authors have found the use of IONM decreased identification time of RLN and operating time [4]. Others have found that neuromonitoring can aid in anatomic identification of RLN and aid in resident training [13-15].

The successful deployment and data analysis from the IONM depend upon the complete laryngeal relaxation and reflex suppression [5]. Laryngeal alterations caused by the strong contractions of the vocal cords, by the IONM have been documented [16]. The surgical procedure is a strong factor of stress to the larynx with potentially traumatic manipulation at the level of the larynx [16]. Complete functionality of the IONM constitutes before full acceleromyographic recovery of the NMBA at the adductor pollicis muscle [17], as the laryngeal muscles recover faster than the peripheral muscles [18]. The pre-curarizing and depolarizing muscle relaxants used in our study were designed to provide adequate relaxation for atraumatic intubation and facilitate Glidescope ${ }^{\circledR}$ visualized electrode encased ETT placement between the vocal cords with the manufacturer recommended alignment. The recovery from the short-acting muscle relaxants was confirmed with the TOF monitor prior to the first IONM stimulation.

The anesthetic technique, either total intravenous anesthesia (TIVA) or narcotic-based inhalational anesthesia (NBIA) should ensure continued alignment of IONM electrodes with the vocal cords throughout the procedure to ensure the integrity of the data output from the monitor. Such data can alter the intraoperative course and postoperative outcome. While muscle relaxants cannot be employed for fear of compromising the IONM data, the frequent intra-laryngeal alterations promoted by the IONM activation and extra-laryngeal surgical manipulations demand a considerable depth of anesthesia to keep the troublesome reflexes at bay. Profound cardiovascular instability can be the result of pursuing such a course. Attempt to counter the instability with sympathomimetic agents can themselves result in undesirable hemodynamic consequences. $\mathrm{Pa}$ tients with significant co-morbidities (ASA III) can ill-afford such an intraoperative course.

Injected intravenously, lidocaine is variably effective in blunting the hemodynamic response to intubation [19]. IV lidocaine decreases the intracellular $\mathrm{Ca}^{2+}$ concentration in the airway smooth muscle, decreases myofilament $\mathrm{Ca}^{2+}$ sensitivity and has been shown to suppress coughing and prevent reflex bronchoconstriction [19]. Laryngoscopy with or without intubation provokes a sympathoadrenal response. These events are clearly detrimental in individuals who have limited myocardial reserve because of age or disease [20]. Administration of lidocaine IV $1.5 \mathrm{mg} / \mathrm{kg}$ attenuates the hyperdynamic response to laryngoscopy and intubation [20].

Because of its plasma half-life of $8 \mathrm{~min}$, an IV bolus of $1-1.5 \mathrm{mg} / \mathrm{kg}$ lidocaine must be followed by infusion of 1 $4 \mathrm{mg} / \mathrm{min}$ in adults in order to maintain therapeutic levels of $1.5-4 \mu \mathrm{g} / \mathrm{mL}[21,22]$. Lidocaine side effects have never been reported in its intended use, probably because the first clinical signs of toxicity occur at considerably high blood concentrations $(>5 \mu \mathrm{g} / \mathrm{mL})$ which did not occur even when IV lidocaine was given continuously over 14 days [23]. Lidocaine infusion at the rate of $1.5 \mathrm{mg} / \mathrm{kg} / \mathrm{h}$ following a bolus of $1.5 \mathrm{mg} / \mathrm{kg}$ has been employed in neonatal neurosurgery to facilitate an intraoperative wake-up test [24].

Visualization of the RLN is the main factor ensuring nerve preservation [12]. The specificity and sensitivity of the IONM can be vastly improved by stimulating the visualized RLN. The optimal output from the IONM depends on the near perfect deployment and the near ideal intra-laryngeal environment. We believe that IVLI in our study has been able to provide good operating and monitoring conditions as well as stable hemodynamic parameters with least side effects especially in ASA III patients.

Suppression of laryngotracheal reflexes during thyroid and parathyroid surgery has largely been monitored by clinical signs like coughing, bucking on the ETT or swallowing under anesthesia. The IONM causes strong contractions of the vocal cords and can cause laryngeal alterations [5]. Such laryngeal alterations may not be clinically discernable, nevertheless contribute to tiny extrusion of the ETT held between the vocal cords. While not strong enough to precipitate extubation, they do cause malalignment of the electrode positioning in the larynx resulting in weaker signal strength and DAIL during the surgery.

Our primary outcome measure results indicated a more stable laryngotracheal environment intraoperatively in the LG compared to the PG. The IVLI used in our study provided us with the superior laryngeal quiescence required to provide the enhanced laryngeal relaxation and reflex suppression. This in turn ensured the maintenance of contact between the electrodes and vocal cords more optimally and for prolonged duration, notwithstanding the intra-laryngeal alterations by the IONM and the extra-laryngeal manipulations during surgery. Our ability to elicit a positive signal with a $0.5 \mathrm{~mA}$ StMC over the duration of surgery and to ensure the DAIL at $<50 \%$ towards the conclusion of the surgery was statistically significant in the LG.

While none of our secondary outcome measures rise to the level of statistical significance, we are encouraged that our exploratory outcome results offered us positive data for future clinical pursuit. The total duration of surgery, additional doses of cisatracurium required intraoperatively, the diastolic pressure on extubation and hypotensive episodes requiring rescue medications more than twice during surgery were all lower in the LG. We believe IVLI has contributed to these results and propose further targeted study.

Rising intolerance for physician error, potential legal issues and medical insurers can increase intraoperative stress for the providers [4]. With respect to thyroid and parathyroid surgery, IONM has largely mitigated such stress among established providers and a whole new generation of trainees. By employing IVLI to improve the functionality of the IONM, the output data can be interpreted with greater confidence thereby minimizing the operating room stress and enhancing the postoperative patient outcome. 


\section{Financial Disclosure}

None to declare.

\section{Conflict of Interest}

None to declare.

\section{Informed Consent}

Written informed consent was obtained from each of the patients during the preoperative interview on the day of the surgery.

\section{Author Contributions}

Dr. Ramasamy Govindarajan was the principal investigator conceptualized the idea and design, conducted the study, and contributed towards the study protocol, anesthesia protocol, IRB submission, clinical trial registration, manuscript preparation, and submission. Dr. Ajay Shah contributed towards the patient recruitment, study protocol, informed consenting, surgical protocol, surgical management and overall study co-ordination. $\mathrm{Mr}$. Saiganesh Ravikumar contributed towards the literature search, informed consenting, preoperative patient counseling, IRB application, statistical analysis, and writing methods and results of the manuscript. Dr. Sunil K. Reddy contributed towards literature search, study design, IRB application, clinical trial registration, surgical protocol, surgical management, and data collection. Dr. Umashankkar Kannan contributed towards IRB application, surgical protocol, surgical management, informed consenting, and data collection. Dr. Amar Mukerji contributed towards the literature search, informed consenting, surgical protocol, surgical management, and data collection. Dr. Jasmine Cherian contributed towards randomization, coding, preparation and delivery of study medication. Dr. Crista Foster contributed towards anesthesia protocol, anesthesia management, informed consenting, and data collection. Dr. Dave Livingstone contributed towards the study design, coordination, informed consenting and anesthesia protocol. All authors discussed the results, provided critical review with feedback and help to shape the conduct of the study and the manuscript. All authors agreed to the final version and accountability to all aspects of the work.

\section{Data Availability}

The authors declare that the data supporting the findings of this study are available within the article.

\section{Author Note}

This study was presented in part at The European Society for Surgical Research (ESSR), 52nd International Meeting, Am- sterdam, June 2017, Best Clinical Research Award Session as an oral presentation by the author Saiganesh Ravikumar.

\section{References}

1. Thomusch O, Sekulla C, Walls G, Machens A, Dralle $\mathrm{H}$. Intraoperative neuromonitoring in surgery for benign goiter: decreased rate of recurrent laryngeal nerve palsy after subtotal thyroidectomy. AM J Surg. 2002;183:674679.

2. Timmermann W, Dralle H, Hamelmann W, Thomusch O, Sekulla C, Meyer T, Timm S, et al. [Does intraoperative nerve monitoring reduce the rate of recurrent nerve palsies during thyroid surgery?]. Zentralbl Chir. 2002;127(5):395-399.

3. Thomusch O, Sekulla C, Machens A, Neumann HJ, Timmermann W, Dralle H. Validity of intra-operative neuromonitoring signals in thyroid surgery. Langenbecks Arch Surg. 2004;389(6):499-503.

4. Sari S, Erbil Y, Sumer A, Agcaoglu O, Bayraktar A, Issever H, Ozarmagan S. Evaluation of recurrent laryngeal nerve monitoring in thyroid surgery. Int J Surg. 2010;8(6):474-478.

5. Govindarajan R, Shah A, Reddy VS, Parithivel V, Ravikumar S, Livingstone D. Improving the functionality of intra-operative nerve monitoring during thyroid surgery: is lidocaine an option? J Clin Med Res. 2015;7(4):282285.

6. Flisberg K, Lindholm T. Electrical stimulation of the human recurrent laryngeal nerve during thyroid operation. Acta Otolaryngol Suppl. 1969;263:63-67.

7. Ho Y, Carr MM, Goldenberg D. Trends in intraoperative neural monitoring for thyroid and parathyroid surgery amongst otolaryngologists and general surgeons. Eur Arch Otorhinolaryngol. 2013;270(9):2525-2530.

8. Marusch F, Hussock J, Haring G, Hachenberg T, Gastinger I. Influence of muscle relaxation on neuromonitoring of the recurrent laryngeal nerve during thyroid surgery. Br J Anaesth. 2005;94(5):596-600.

9. Bergamaschi R, Becouarn G, Ronceray J, Arnaud JP. Morbidity of thyroid surgery. Am J Surg. 1998;176(1):71-75.

10. Sturniolo G, D'Alia C, Tonante A, Gagliano E, Taranto F, Lo Schiavo MG. The recurrent laryngeal nerve related to thyroid surgery. Am J Surg. 1999;177(6):485-488.

11. Perie S, Ait-Mansour A, Devos M, Sonji G, Baujat B, St Guily JL. Value of recurrent laryngeal nerve monitoring in the operative strategy during total thyroidectomy and parathyroidectomy. Eur Ann Otorhinolaryngol Head Neck Dis. 2013;130(3):131-136.

12. Randolph GW, Kobler JB, Wilkins J. Recurrent laryngeal nerve identification and assessment during thyroid surgery: laryngeal palpation. World J Surg. 2004;28(8):755760.

13. Chan WF, Lang BH, Lo CY. The role of intraoperative neuromonitoring of recurrent laryngeal nerve during thyroidectomy: a comparative study on 1000 nerves at risk. Surgery. 2006;140(6):866-872; discussion 872-863.

14. Hermann M, Hellebart C, Freissmuth M. Neuromoni- 
toring in thyroid surgery: prospective evaluation of intraoperative electrophysiological responses for the prediction of recurrent laryngeal nerve injury. Ann Surg. 2004;240(1):9-17.

15. Loch-Wilkinson TJ, Stalberg PL, Sidhu SB, Sywak MS, Wilkinson JF, Delbridge LW. Nerve stimulation in thyroid surgery: is it really useful? ANZ J Surg. 2007;133:481485.

16. Birkholz T, Irouschek A, Saalfrank-Schardt C, Klein P, Schmidt J. Laryngeal morbidity after intubation with or without neuromuscular block in thyroid surgery using recurrent laryngeal nerve monitoring. Auris Nasus Larynx. 2012;39(3):288-293.

17. Chu KS, Wu SH, Lu IC, Tsai CJ, Wu CW, Kuo WR, Lee $\mathrm{KW}$, et al. Feasibility of intraoperative neuromonitoring during thyroid surgery after administration of nondepolarizing neuromuscular blocking agents. World J Surg. 2009;33(7):1408-1413.

18. Hemmerling TM, Donati F. Neuromuscular blockade at the larynx, the diaphragm and the corrugator supercilii muscle: a review. Can J Anaesth. 2003;50(8):779-794.

19. Carpenter RL, MacKey D. Local anesthetics. In: Barash PG, Cullen BF, Stoelting RK, eds. Clinical anesthesia. Philadelphia. Lippincott Raven. 1997; p. 434.

20. Mallampati RS. Airway management. In: Barash PG, Cullen BF, Stoelting RK, eds. Clinical anesthesia. Philadelphia. Lippincott Raven. 1997; p. 587.

21. Nadrowski L. Paralytic ileus: recent advances in pathophysiology and treatment. Curr Surg. 1983;40(4):260-273.

22. Ness TJ. Intravenous lidocaine inhibits visceral nociceptive reflexes and spinal neurons in the rat. Anesthesiology. 2000;92(6):1685-1691.

23. Williams DR, Stark RJ. Intravenous lignocaine (lidocaine) infusion for the treatment of chronic daily headache with substantial medication overuse. Cephalalgia. 2003;23(10):963-971.

24. Govindarajan R, Babalola O, Gad-El-Kareem M, Kodali NS, Aronson J, Abadir A. Intraoperative wake-up test in neonatal neurosurgery. Paediatr Anaesth. 2006;16(4):451453. 